GRADIATION\&APPLICATIONS

ISSN 2466-4294 (online) | rad-journal.org

Vol. 3 | Issue 2 | pp. 143-146, 2018

doi: 10.21175/RadJ.2018.02.024

Original research paper

\title{
VISUALIZATION AND MORPHOLOGICAL CHARACTERIZATION OF INTEGRAL SKIN CELLULAR POLYMERIC COMPOSITES USING X-RAY MICROTOMOGRAPHY*
}

\author{
R. Pop-Iliev**, W. Y. Pao, P. Karimipour-Fard, G. Rizvi \\ University of Ontario Institute of Technology, Oshawa, ON, Canada
}

\begin{abstract}
This paper focuses on 3-dimensional non-destructive characterization of the morphologies of integral-skin cellular polymeric composites using X-ray Microtomography. Rapid Rotational Foam Molding (RRFM) is a polymer processing technology that is capable of creating composites with intricate shapes that have a foamed core surrounded by an integral solid skin layer (similar to the structure of a bone). The analyzed specimens were extracted from composites processed in RRFM having a solid skin made of polypropylene (PP) grades combined with foamed cores made of both polyethylene (PE) and PP grades by implementing a suitable chemical blowing agent (CBA) in extrusion. The resulting cellular structures pertaining to the foamed core and the near-skin area were visualized and their morphological quality was evaluated in terms of cell size distribution and cell density. The stress-strain behavior and 3-dimensional structural changes were monitored and characterized with in-situ compression testing.
\end{abstract}

Key words: Cellular properties, in-situ compression, integral-skin cellular composite, Micro-Computed Tomography, Rapid Rotational Foam Molding

\section{INTRODUCTION}

Integral-skin cellular polymeric composites are made of a solid skin and a foamed polymeric core, such as polyethylene (PE) and polypropylene (PP). The composite products are originally nature inspired by bones. Bone is a natural composite structure with characteristics of high stiffness and tensile strength [1]. High mechanical strength, high porosity, efficient pore interconnectivity, and graded pore size [2]. Cellular structures having mean pore size of 200 to $500 \mu \mathrm{m}$ are considered as adequate for potentially achieving a high strength-to-density ratio [2]. The structure of bone because of its high mechanical strength per volume is therefore an inspiration to manufacture foam/skin composites by Rapid Rotational Foam Molding (RRFM) technique.

RRFM is a patented manufacturing technique that is capable of producing composite with high porosity and interconnected pores in micro scale [3, 4]. RRFM process combines biaxial rotational molding of solid skin with extrusion foaming of the core. The process is continuous where the skin is not cooled during the procedure in order to achieve strong physical bondings between the skin and foam interfaces [3, 4]. The RRFM technique is also capable of producing functionally graded foam from the skin to the core; smaller pore size near the skin and larger pore size at the core. The performance of the foamed core can be evaluated based on its compressive strength, which is affected by cellular properties including cell size, cell density, and cell wall thickness. There are many characterization techniques, among them X-ray micro-computed tomography is a suitable method that is non-destructive and is capable of depicting both qualitative visualization and quantitative analysis.

Micro-CT is a technique which uses a series of $\mathrm{x}$-ray transmission images acquired at different angles to generate a $3 \mathrm{D}$ reconstruction of the sample. The quality of an image, can be altered by the accelerating voltage of energy, and current of X-rays; the intensity of X-ray is therefore proportional to the product of voltage, current, and exposure time. [5]

This paper focuses on the 3-dimensional morphological and mechanical studies of PE and PP foams extracted from integral-skin cellular products. The relationship between cellular properties and compressive strength were evaluated using X-ray micro-computed tomography. This paper intends to provide insight in the use of polymeric composite materials in the biomedical field.

\section{EXPERIMENTAL}

RRFM manufacturing technique was employed to produce the integral-skin cellular composites made of PP skin and two different grades of each PE and PP foams, including linear low-density polyethylene (LLDPE), single site catalyzed high density polyethylene (sHDPE) [6], homopolymer polypropylene (h-PP), and copolymer polypropylene (c-PP). Grades of polymers were differentiated by the compositions and melt flow rates (MFR) and are shown in Table 1 . Chemical blowing agents Celogen OT and Celogen AZ were used to assist extrusion foaming, volume expansion ratios of six and four were achieved for PE and PP, respectively.

\footnotetext{
* This paper was presented at the Sixth International Conference on Radiation and Applications in Various Fields of Research (RAD 2018), Ohrid, Macedonia, 2018.

*** remon.pop-iliev@uoit.ca
} 
R. Pop-Iliev et al., Visualization and morphological characterization..., Rad. Applic., 2018, 3, 2, 143-146

Table 1. Polymer material properties

\begin{tabular}{|c|c|c|c|}
\hline Material & Part & $\begin{array}{c}\text { MFR } \\
(\mathrm{g} / 1 \mathrm{omin})\end{array}$ & $\begin{array}{c}\text { Density } \\
\left(\mathrm{g} / \mathrm{cm}^{3}\right)\end{array}$ \\
\hline PP & Skin & 8 & 0.9 \\
\hline LLDPE & Foam & 3.6 & 0.94 \\
\hline sHDPE & Foam & 1.7 & 0.97 \\
\hline h-PP & Foam & 4 & 0.9 \\
\hline c-PP & Foam & 2 & 0.902 \\
\hline
\end{tabular}

Micro-computed tomography was employed to characterize the integral-skin cellular composite specimens prepared (precisely cut) from a region adjacent to the skin.

An X-ray Micro-CT scanner Skyscan 1172 from Bruker was used to scan cellular structures. To scan the cellular structure with high resolution, it is necessary to perform flat field correction. During the flat field correction procedure, the voltage of the Micro-CT scanner was changed through multiple iterations to find out the optimal contrast between the background and the sample. The optimal voltage and current for the scanned samples were determined to be $54 \mathrm{kV}$ and $71 \mu \mathrm{A}$ for all scans.

With respect to the imaging procedure, a resolution of $2000 \mathrm{x} 1000$ pixels with size of $12 \mu \mathrm{m}$ was set for all of the cellular structures. $360^{\circ}$ scans with rotational steps of $0.6^{\circ}$, and frame averaging of 7 were used to achieve the best results for all areas of the samples.

NRecon, CTAn, and CTVol software packages were used for image post-processing [7]. NRecon software was employed to reconstruct the $3 \mathrm{D}$ structure of the scanned images. Next, the CTAn software was used to assess the morphological properties of the cellular structures in 3D. Thresholding and despeckling procedures were performed before the $3 \mathrm{D}$ analysis to achieve reliable morphological results (porosity analysis). As a result, 3D models with phase separations of cell walls, open cells, and closed cells were obtained using the CTVol software.

An in-situ mechanical test stage was used for compression stress-strain tests (MTS1 Stage). The main advantage of this mechanical test stage is the opportunity to utilize the micro-computed tomography technique to monitor the changes in morphology and mechanical properties of the samples during compression. The cellular structure was preloaded by 3 $\mathrm{N}$ force before the compression test and morphological monitoring of the samples were done in three points including before the compression, at $30 \%$ strain and at the $50 \%$ strain (the end of the compression test).

\section{RESULTS AND DISCUSSION}

\subsection{Morphology}

Both 2D and 3D morphologies are presented in Figure 1 and 2, generated from CTAn and CTVol, respectively. There were distinctive differences between $\mathrm{PE}$ and PP foams in terms of cell shape and cell wall thickness.

PE foams were observed to have irregular cell shapes whereas PP foams were found to have spherical-like shapes as seen in Figure 1. This could be due to the difference in melt strength of the two materials which defines foamability.

PP foams were also observed to have thicker cell walls than PE foams as seen from the white regions which denotes material, and black corresponds to pores (cells). Interconnectivity in $3 \mathrm{D}$ models: LLDPE has much thinner cell walls, and most structures are smaller open cells, therefore having a higher connectivity than h-PP.

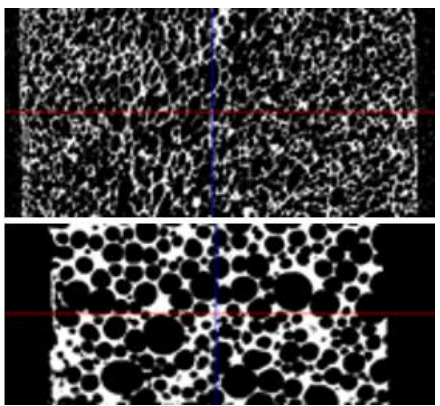

Figure 1. 2D LLDPE and h-PP foam structures

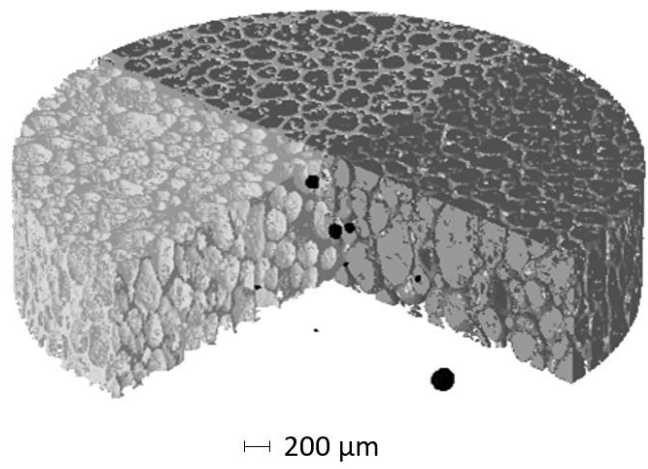

(a)

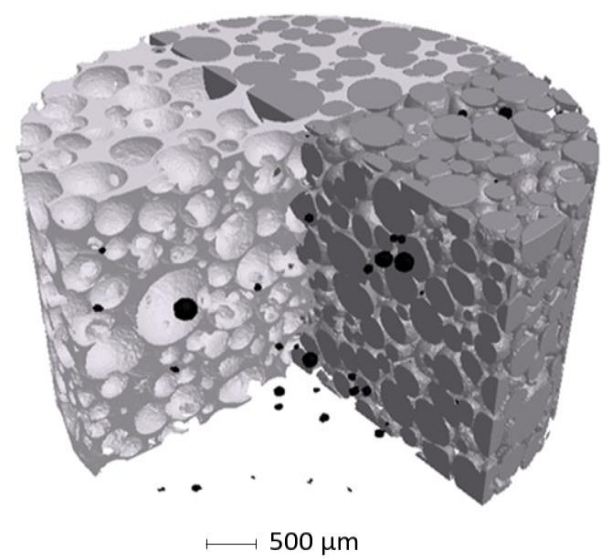

(b)

Figure 2. 3D model of (a) LLDPE and (b) h-PP foam structures showing cell walls (light grey), interconnected open cells (dark grey), and closed cells (black)

\subsection{Cellular properties}

Quantitative cellular properties including cell size distribution, cell density, cell wall thickness, and percent porosity were evaluated using $3 \mathrm{D}$ analysis by 
CTAn software. The cellular properties of the integral cellular composite products were examined near the skin-foam region, and the results are presented in Table 2.

In general, PE foams were found to have smaller average cell size and wall thickness, but higher percent porosity than those of PP foams. The porosity recorded for $\mathrm{PE}$ foams range from $70-80 \%$ while $\mathrm{PP}$ varies between $55-65 \%$.

Figure 3 shows cell size distributions for the specimens evaluated. LLDPE was observed to have the narrowest range of cell sizes thus the high cell density, whereas SHDPE had the widest distribution due to cell coalescence during the manufacturing process. All foams showed a unimodal behavior, and most of them showed asymmetric distribution except for h-PP. The larger average cell size in h-PP compared to c-PP could be a result of the difference in MFR, which affects the cell growth during the manufacturing process.

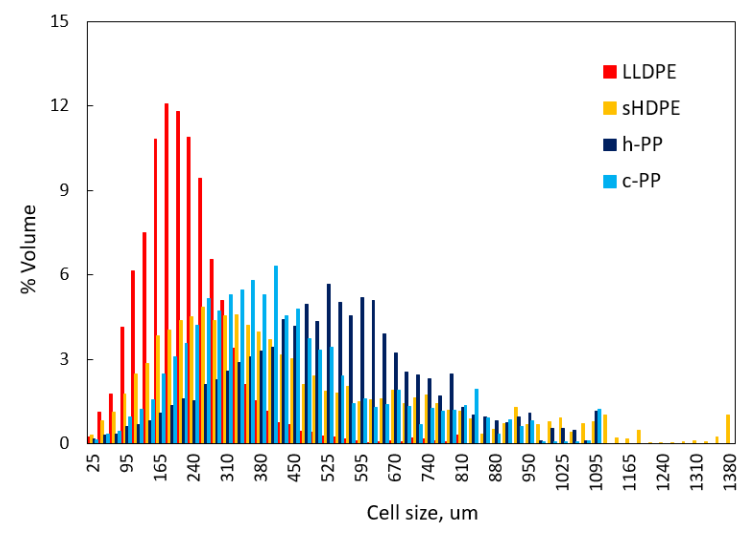

Figure 3. Cell size distributions of PE and PP foams

Table 2. Cellular properties

\begin{tabular}{|c|c|c|c|c|c|}
\hline Material & $\begin{array}{c}\text { Avg cell } \\
\text { size }(\mu \mathrm{m})\end{array}$ & $\begin{array}{c}\text { Max cell } \\
\text { size }(\mu \mathrm{m})\end{array}$ & $\begin{array}{c}\text { Cell } \\
\text { density } 10^{4} \\
\left(\text { cells } / \mathrm{cm}^{3}\right)\end{array}$ & $\begin{array}{c}\text { Avg wall } \\
\text { thickness } \\
(\mu \mathrm{m})\end{array}$ & $\begin{array}{l}\text { Max wall } \\
\text { thickness } \\
(\mu \mathrm{m})\end{array}$ \\
\hline LLDPE & 190 & 810 & 90.6 & 50 & 285 \\
\hline SHDPE & 260 & 1380 & 31.7 & 50 & 240 \\
\hline h-PP & 525 & 1095 & 14 & 170 & 475 \\
\hline c-PP & 405 & 1095 & 3.98 & 190 & 405 \\
\hline
\end{tabular}

\subsection{Mechanical tests}

The results of uniaxial compression tests are presented as stress-strain curves in Figure 4. PP foams were observed to have higher compressive strength than $\mathrm{PE}$ foams, by a magnitude of $3 \mathrm{MPa}$. The huge difference is due to the molecular structures of the two polymeric materials, where a lower crystallinity nature of PP was observed to result in stronger strength. The shape of cellular structures could also be another factor, where a more spherical and elliptical shape in PP would resist compression more than a random polygonal shape in PE.

There were two transitions throughout the compression until $50 \%$ strain, the first one transitioned from a higher to lower slope assuming bi-linear behavior, the second one transitioned with an almost exponential growth of stress. The first transition is determined to be the compressive strength of the material where cell walls begin to collapse. The second transition occurs when the foam densifies to become solid-like and require higher stress to further compress the material. The two transitions can be visualized in Figure 5 .

During the compression, X-ray scans were performed at $30 \%$ and $50 \%$ strain for a selected PE and PP specimen. Quantitative results suggested that cell size reduces and cell density increases with compression.

Morphological analysis illustrated that mechanical strength is a function of cellular properties. For PE foams (grey), smaller cell size and higher cell density in LLDPE resulted in higher compressive strength than sHDPE by 0.2 MPa. For PP foams (black), thicker cell walls and higher cell density in h-PP resulted in higher stress required in compression than c-PP.

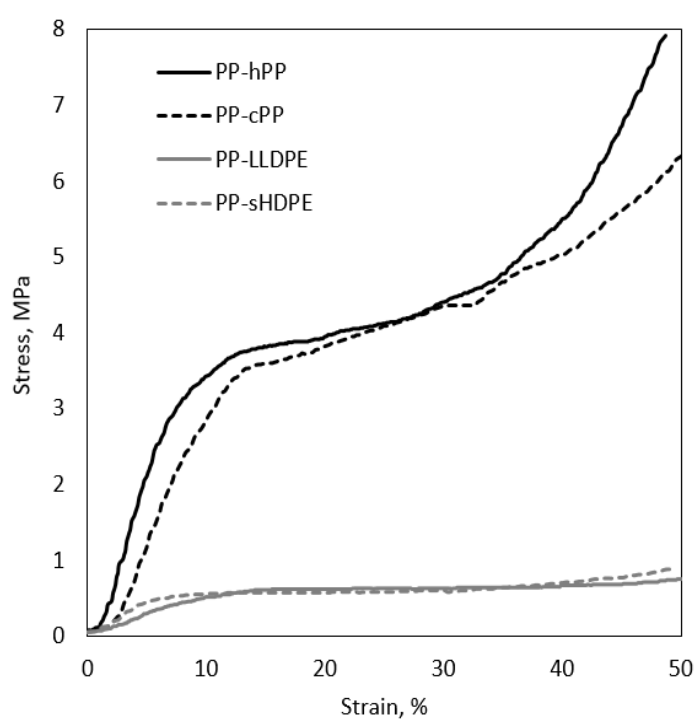

Figure 4. Compression stress-strain curves

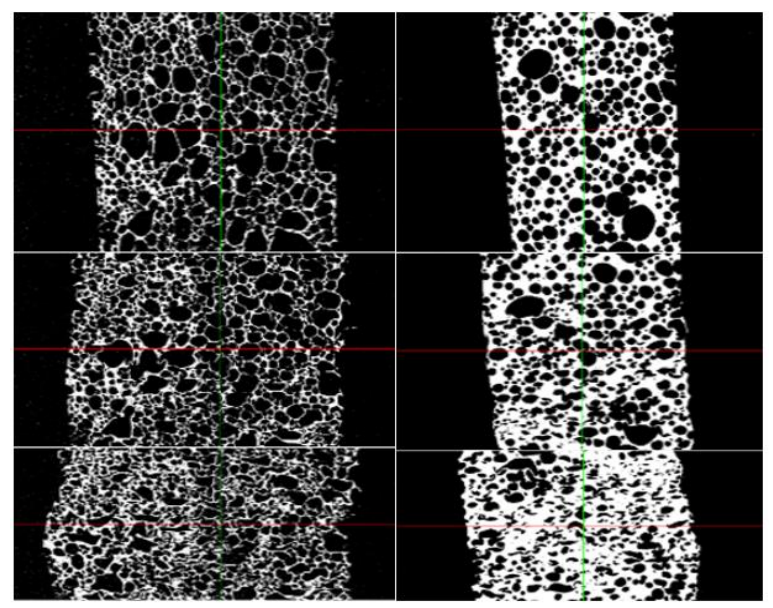

(a) PP Skin - sHDPE Foam

(b) PP Skin - c-PP Foam

Figure 5. Morphology change during compression at o, 30, and $50 \%$ strain for (a) sHDPE foam and (b) c-PP foam 


\section{CONCLUSION}

In this study, Rapid Rotational Foam Molding manufacturing method was used to manufacture skin/foam integral composites that have some crucial properties of bone structure to produce a light structure with high strength. Microcomputed tomography technique and in-situ mechanical compression test were used to characterize the produced composite's cellular structures near the skin. Morphological analysis was conducted for all samples during the mechanical test to assess the behavior of cells under mechanical compression test.

The obtained experimental results could be summarized as follows:

- By comparison, the analyzed PP foam morphologies demonstrated a significantly higher presence of spherical cells and thicker cell walls than those of PE foamed structures. This fact indicates that PP foams would be expected to have superior mechanical strength properties.

- Mechanical strength of PP foam is much higher than that of PE foams, which is mostly because of the materials properties, but the orderly disturbed spherical structures of the cells could have a share in this high difference.

- With respect to cell interconnectivity, LLDPE foams demonstrated the highest degree of interconnectivity in comparison to the other samples and would thus be preferred in applications that interconnectivity is needed.

- As expected, h-PP cellular structures showed higher strength than c-PP because of the intrinsically superior morphological properties.

- Bone tissue structure characteristics were the incentive to use RRFM for manufacturing of skin/foam composites; the results have shown that higher porosity and interconnectivity of cellular structures (LLDPE foam) would reduce mechanical strength, and on the other hand, wellshaped spherical cellular structures and thicker cell walls would increase mechanical strength (hPP foam).

Therefore, achieving an optimal balance between the above-described factors would be the best strategy to follow with a view of producing artifacts that would have high mechanical strength and low weight.
Acknowledgement: The authors are grateful to the financial support provided by NSERC's Discovery Development Grant and NSERC's Chairs in Design Engineering Program. The authors would also like to acknowledge the material supply by NOVA Chemicals Inc. and LyondellBasell.

\section{REFERENCES}

1. P. Bianco, M. Riminucci, S. Gronthos, P. Gehron, "Bone marrow stromal stem cells: nature, biology, and potential applications," Stem Cells, vol. 19, no. 3 pp. $180-192,2001$.

DOI: $10.1634 /$ stemcells.19-3-180 PMid: 11359943

2. V. Karageorgiou, D. Kaplan, "Porosity of 3D biomaterial scaffolds and osteogenesis," Biomaterials, vol. 26 , no. 27, pp. $5474-5491$, Sep. 2005. DOI: 10.1016/j.biomaterials.2005.02.002 PMid: 15860204

3. R. Pop-Iliev, "Processing of Integral Skin Cellular Polymeric Composites in Rapid Rotational Foam Molding," Acta Phys. Pol. A, vol. 120, no. 2, pp. $292-297,2011$. DOI: 10.12693/APhysPolA.120.292

4. R. Pop-Iliev, K. A. Christian, E. S. Abdalla, "Rapid Rotational Foam Molding Process," US 8628704 B2, USA, Jan. 14, 2014 Retrieved from: https://patentimages.storage.googleap is.com/2d/40/f5/63d6a3b37d3cao/US8628704.pdf; Retrieved on: Jun. 24, 2017

5. K. Holmes, M. Elkington, P. Harris, "Clark's Essential Physics in Imaging for Radiographers," Boca Raton (FL), USA: CRC Press, ch. 5-6, sec. 3-14, pp. 68 - 90, 2013. DOI: $10.1201 / \mathrm{b} 15383$

6. N. Aubee, P. Lam, S. Marshall, "A New Family of sHDPE Polymers for Enhanced Moisture Barrier Performance," J. Plast. Film Sheeting, vol. 22, no. 4, pp. 315 - 330, Oct. 2006. DOI: $10.1177 / 8756087906073121$

7. P. Karimipour-Fard, W. Y. Pao, G. Rizvi, R. Pop-Iliev, "The Use of Microcomputed Tomography to Evaluate Integral-Skin Cellular Polyolefin Composites," in Book of Abstr. Micro-CT Meeting 2018 (Micro-CT 2018), Ghent, Belgium, 2018, pp. $41-44$.

Retrieved from: https://www.bruker.com/fileadmin/us er upload/8-PDFDocs/Microtomography/UserMeeting/AbstractBook20 18.pdf;

Retrieved on: Jun. 17, 2018 